\title{
Editorial
}

Joanna Sheldon

\section{Developments in laboratory testing for autoimmune diseases}

https://doi.org/10.1515/cclm-2018-0233

There are at least 100 different autoimmune diseases that can affect any organ of the body. In the USA, it is estimated that autoimmune diseases affect between 5\% and $8 \%$ of the population (14.7-23.5 million people), but individual autoimmune diseases like Addison's disease, autoimmune neuropathies and ACNA vasculitis can certainly be classified as rare (affecting less than 200,000 people [USA] or fewer than 1 in 2000 of the population [Europe]). Nevertheless, autoimmune diseases often require lifelong treatment, are considerably more prevalent among women and are among the 10 leading causes of mortalilty in women under 65 years. It is also interesting to compare the prevalence of autoimmune diseases to, for example, heart disease (22\%) and cancer (9\%), which seem to have a much higher public profile [1] and research support with funding quoted in billions of dollars rather than the millions for autoimmune diseases. The incidence of autoimmune diseases is increasing. For example, the International Diabetes Atlas reported that in 2017 there were 10 million more adults worldwide with type I diabetes that in 2015, and if this trend continues, by 2045 , 67 million Europeans will be living with type I diabetes [2].

Over the last 20 years, our ability to detect and quantify the antibodies involved in autoimmune diseases has improved, and gradually such tests are becoming incorporated onto high throughput platforms often in Clinical Blood Sciences. However, autoantibody measurements can lack clinical specificity and sensitivity and show high variation between platforms and tests, and certified reference materials are only recently available for only a small number of autoantibodies. The number of requests is increasing far beyond what would be expected for a tests for rare conditions and with low pretest probabilities. Numerical reports give no impression of the "uncertainty" of the result, and the enthusiasm for automated analysis and reporting puts the responsibility on the requesting clinician to interpret the result, often without understanding of the limitations of the test.

This issue of Clinical Chemistry and Laboratory Medicine highlights the diversity of autoimmune disease from organ-specific conditions to systemic multiorgan disease. It reminds us that there is significant overlap between Clinical Chemistry and Immunology and that we have an important role the diagnosis and management of a variety of autoimmune disease. We can clearly see how much work is being done to investigate, understand and improve autoantibody testing, but there is still much to be done to optimise the requesting, the analytical process and the interpretation of these complex assays.

More than 90\% of cases of Addison's disease have an autoimmune aetiology with antibodies to adrenal tissue and more specifically antibodies to 21-hydroxylase being markers of the disease. The symptoms of adrenal insufficiency can be subtle, but patients may also present as an emergency in Addisonian crisis. It is also well known that Addison's is associated with other autoimmune endocrinopathies, e.g. Grave's disease, hypothyroidism or type I diabetes. Manso et al. [3] describe a patient with autoimmune hypothyroidism where the patient's family history led to the finding of positive anti-adrenal antibodies. Further investigation confirmed the diagnosis of subclinical Addison's and treatment was started. Their literature review showed that only $1 \%$ of patients with autoimmune thyroid disease had anti-adrenal-cortex antibodies. Use of a more sensitive ELISA-based assay increased the detection of antibodies to 21-hydroxylase in children with autoimmune thyroid disease to approximately 4 percent, although even at this frequency, it would only be worth checking for anti-adrenal antibodies in patient with clinical features or family history. Surprisingly, anti-adrenal-cortex antibodies are found in a much greater proportion of patients with premature ovarian failure and in chronic hypoparathyroidism, so screening for antibodies to 21-hydroxylase in such patients may be useful. Classically, adrenal antibodies have been detected by indirect immunofluorescence. This can be technically demanding, and the interpretation of the staining needs considerable training and experience. del Pilar Larosa et al. [4] have reported the development of an ELISA for 21-hydroxylase, the enzyme, which is the important antigen within the adrenal cortex. They report good specificity and sensitivity for Addison's disease so this may be 
a better method for supporting a diagnosis of Addison's than the qualitative immunofluorescence techniques.

Type I diabetes also has an autoimmune aetiology, and in patients where the clinical and biochemical presentation is complex, antibodies to insulin, glutamic acid decarboxylase (GAD), insulinoma antigen (IA2) and zinc transporter 8 (ZnT8) may be measured to improve the diagnostic power. Censi et al. [5] report the case of a patient with high blood insulin concentrations, hypoglycaemia and a high serum concentration of antibodies to insulin. It is important that we are aware of these antibodies because they loosely bind to insulin, which is gradually released independent of the normal feedback mechanism generating hypoglycaemia. These circulating anti-insulin autoantibodies also have the capacity to interfere in analyses that may be used in the investigation of unexplained hypoglycaemia, e.g. insulin, C-peptide and proinsulin immunoassays.

There has been a dramatic improvement in the understanding of autoimmune mediated neuropathies over the last 20 years with autoantibody responses to gangliosides being implicated in their pathology. These conditions can be acute or chronic; they can affect the motor, sensory or autonomic nerves; and the presenting features can be variable. Improved diagnosis with prompt treatment could prevent irreversible nerve damage and consequent disability. Klehmet et al. [6] describe a multiplex approach to detecting antibodies IgG and IgM antibodies to 11 ganglioside antigens in patients with autoimmune neuropathies, other neurological conditions and normal subjects. The IgM anti-sulphatyl, IgM anti-GM1 and IgM anti-GD1b antibodies showed a higher frequency in the inflammatory neuropathy patients compared to patients with other neurological conditions. This is early data, and the authors suggest that further investigation is warranted, but it does show the power of multiplex-type analysis in identifying markers for further directed investigation.

The term "phospholipid" antibodies like the term "neurological" antibodies describes antibodies to a number of distinct antigenic targets. In both situations, the tissues where these antigens are expressed are throughout the body generating widespread symptoms. Phospholipids, including cardiolipin and $\beta_{2}$ glycoprotein 1 , phosphatidylcholine, phosphatidylethanolamine, phosphatidylserine and phosphatidylinositol, are an integral part of all cell membranes. Antibodies to these components are associated with arterial or venous thrombosis. There are welldefined diagnostic criteria for antiphospholipid syndrome, but only measurements of IgG and IgM antibodies to cardiolipin and/or $\beta_{2}$ glycoprotein 1 are included alongside lupus anticoagulant (an adaptation of a clotting test). Antibodies to the other phospholipids have generally been considered to have limited diagnostic value; however, Zhang et al. [7] have evaluated the clinical relevance of antibodies to a phosphatidylserine-prothrombin complex. They found increased concentrations of IgG and IgM antibodies to phosphatidylserine-prothrombin complex in their patients with antiphospholipid syndrome, although in their study, cardiolipin antibodies showed the best clinical performance. Interestingly, antibodies to the phosphatidylserine-prothrombin complex were detected in patients with clinical features of antiphospholipid syndrome, but negative antibodies to the classical antigens, suggesting that although the majority of patients show cardiolipin antibodies, other antigenic specificities may be important in individual patients.

Antinuclear antibodies (ANAs) is a commonly requested autoantibody with importance in the diagnosis of systemic autoimmune rheumatic diseases such as systemic lupus erythematosus, Sjogren syndrome, etc. ANAs have classically been detected by a manual indirect immunofluorescence method, which needs good laboratory skills and the ability to recognise the diverse and often complex patterns seen at the microscope reading of the slides. Samples with positive staining patterns have reflex testing by ELISA-based assay for antibodies to specific antigens. The deskilling of laboratories and the simple volume of requests are driving automation for ANA testing. Willems et al. [8] report a review of almost 10,000 patients who were tested for ANA by indirect immunofluorescence and an automated ANA "screen". They report reasonable concordance and comparable receiver operator curves between the two methods. However, simply looking at the comparison does not tell the whole story. The data were also analysed by a requestor, who showed a significantly higher PPV for systemic autoimmune rheumatic diseases when testing was requested by clinicians specialised in systemic rheumatic disease. They concluded that the performance of immunoassay and indirect immunofluorescence depends on the specific disease being considered and that for optimum value both assay types are best used in combination and results interpreted in the context of the clinical picture.

Antibodies to the extractable nuclear antigens Ro/SSA and La/SSB are found in a number of systemic connective tissue diseases, e.g. Sjogren syndrome, systemic lupus erythematosus, etc. These antibodies, if present, cross the placenta from mum to baby as part of the active IgG transport. The Ro and La antigens are expressed in foetal cardiac tissue from the 18th to the 24th week, particularly on the surface of cardiac myocytes, and in a proportion of patients, the antibodies cause inflammation, fibrosis and calcification of foetal cardiac conduction tissues leading 
to atrioventricular block. Antibodies to the p200 epitope of the Ro52/SSA antigen appear to have a critical pathogenic role the development of foetal heart block. Mattia et al. [9] have evaluated the performance of two "in-house" assays for anti-p200 antibodies in pregnant women positive for anti- SSA/Ro52 antibodies. They did find differences in assay performance, but further investigation may identify an analytical process for anti-p-200 antibodies that may help identify patients who are at a significantly increased risk future pregnancy being affected by foetal heart block.

Antineutrophil cytoplasmic antibodies (ANCAs) are autoantibodies that are mainly directed against the cytoplasmic antigens proteinase 3 and myeloperoxidase of neutrophils and the monocytes. Their presence is associated with systemic vasculitides and in particular granulomatosis with polyangiitis, microscopic polyangiitis and Churg-Straus syndrome. Like ANAs, indirect immunofluorescence and ELISA-based assay are used together to detect and quantify these antibodies with a similar drive to replace the qualitative tests with automated quantitative analyses. Certified reference preparations for IgG anti-proteinase 3 and IgG anti-myeloperoxidase are only recently available and are not yet embedded in the analytical process, making comparison of results over time and between methods difficult. Borderline positive results, irrespective of the method used, present the greatest interpretative challenge with limited data on their significance. Watad et al. [10] present a retrospective study of ANCA investigations and conclude that patients with borderlinepositive IgG anti-myeloperoxidase antibodies and positive ANCA staining by indirect immunofluorescence have a poorer outcome in terms of their renal function than patients with negative ANCA staining.

Autoantibodies are complex analytes; the antigens that we use in the analysis vary subtly between companies, and even within a company between different lots, the antibodies that we are trying to detect can vary between patients and even within a patient during the disease course. The demand for autoantibody testing, particularly for the rare autoimmune diseases, is increasing beyond logic, but instead of educating users and optimising demand, we are increasing the capacity for measurement with high throughput automated systems. The analytical speed and detection systems introduce another source of variation into a group of tests where robust standardisation does not exist.

The papers in this issue of Clinical Chemistry and Laboratory Medicine clearly show the relevance of autoantibody testing in a diverse range of disease. However, none of the analytes described in these eight papers were measured with respect to certified reference materials; as a scientific community, we need to embrace standardisation (or harmonisation) for autoantibody testing. Some of these papers also review large amounts of data, e.g. 14,555 ANCA results over a 10-year period [10] and 9856 ANA results over a 2-year period [8]. We consider the approximate incidence and prevalence of ANCA vasculitis (incidence 2/100,000 and prevalence 26/100,000) and SLE (incidence 5/100,000 and prevalence 52/100,000), but we may question why so many of these tests were requested when the pretest probability is so low. We have two enormous challenges - to ensure that test tests are requested appropriately and to improve the analysis and reduce the variability of autoantibody testing.

Author contributions: The author has accepted responsibility for the entire content of this submitted manuscript and approved submission.

Research funding: None declared.

Employment or leadership: None declared.

Honorarium: None declared.

\section{References}

1. Directorate General for Internal Policies, Policy Department A: Economic and Scientific policy. Workshop Autoimmune Diseases - Modern Diseases, Brussels 25th Sep 2017 Proceedings. Available at: http://www.europarl.europa.eu/cmsdata/133620/ ENVI\%202017-09\%20WS\%20Autoimmune\%20diseases $\% 20 \% 20$ PE\%20614.174\%20(Publication).pdf.

2. International Diabetes Federation IDF Diabetes Atlas, 8th ed. Available at: http://www.diabetesatlas.org/resources/2017atlas.html.

3. Manso J, Pezzani R, Scarpa R, Gallo N, Betterle C. The natural history of autoimmune addison's disease with a non-classical presentation: a case report and review of literature. Clin Chem Lab Med 2018;56:896-900.

4. del Pilar Larosa M, Chen S, Steinmaus N, Macrae H, Guo L, Masiero S, et al. A new ELISA for autoantibodies to steroid 21-hydroxylase. Clin Chem Lab Med 2018;56:933-8.

5. Censi S, Albergoni MP, Gallo N, Plebani M, Boscaro M, Betterle C. Insulin autoimmune syndrome (Hirata's disease) in an Italian patient: a case report and review of the literature. Clin Chem Lab Med 2018;56:889-95.

6. Klehmet J, Märschenz S, Ruprecht K, Wunderlich B, Büttner T, Hiemann R, et al. Analysis of anti-ganglioside antibodies by a line immunoassay in patients with chronic-inflammatory demyelinating polyneuropathies (CIDP). Clin Chem Lab Med 2018;56:919-26.

7. Zhang S, Wu Z, Zhang W, Zhao J, Norman GL, Zeng X, et al. Antibodies to phosphatidylserine/prothrombin (aPS/PT) enhanced the diagnostic performance in Chinese patients with antiphospholipid syndrome. Clin Chem Lab Med 2018;56:939-46.

8. Willems P, De Langhe E, Claessens J, Westhovens R, Van Hoeyveld E, Poesen K, et al. Screening for connective tissue 
disease-associated antibodies by automated immunoassay. Clin Chem Lab Med 2018;56:909-18.

9. Mattia E, Hoxha A, Tonello M, Favaro M, Del Ross T, Calligaro A, et al. Detection of autoantibodies to the p200-epitope of SSA/ Ro52 antigen. A comparison of two laboratory assays. Clin Chem Lab Med 2018;56:927-32.

10. Watad A, Bragazzi NL, Sharif K, Gilburd B, Yavne Y, McGonagle D, et al. Borderline positive antineutrophil cytoplasmic antibodies
(ANCA)-PR3/MPO detection in a large cohort tertiary center: lessons learnt from a real-life experience. Clin Chem Lab Med 2018;56:947-53.

Dr. Joanna Sheldon, Protein Reference Unit, St. George's Hospital, Blackshaw Road, SW17 0NH, London, UK,

E-mail: jsheldon@sgul.ac.uk 